\title{
Évaluation de différents paramètres permettant d'estimer la teneur en lipides des chèvres laitières selon leur stade physiologique
}

\author{
P Morand-Fehr, P Bas, P Schmidely, J Hervieu, D Sauvant
}

INRA, station de nutrition et alimentation de I'INA-PG, 16, rue Claude-Bernard, 75231 Paris Cedex 05, France

\begin{abstract}
Summary - Evaluation of various parameters predicting the body lipid content of dairy goats in different physiological states. It was shown in 31 dairy goats, slaughtered in early or mid lactation, or dry and non-pregnant, that body condition scores are a good predictor of body lipid content, and are far better than using live weight. However, as a predictor it is necessary to take into account whether goats are lactating or not.
\end{abstract}

Introduction - Une première étude (Morand-Fehr et al, 1990) a permis d'évaluer différents paramètres en tant qu'estimateurs de la teneur en lipides des chèvres en pleine lactation. En raison des grandes variations des réserves corporelles au cours du cycle gestation-lactation (Chilliard et al, 1987), il était nécessaire de préciser si leur aptitude à estimer cette teneur variait selon le stade physiologique des chèvres.

Matériel et méthodes - Au cours de 3 années successives, 31 chèvres Alpines et Saanen âgées de plus d' 1 an ont été abattues à 3 stades différents : 11 chèvres sont abattues entre la $7^{*}$ et la $9^{\circ}$ semaine de lactation, 10 autres entre la $11^{e}$ et la $19^{e}$ semaine et les 10 dernières, non gestantes et taries depuis 5-7 semaines. Auparavant, elles ont reçu une ration à base de foin de luzerne, de pulpes de betteraves ensilées et d'aliments concentrés. Pendant les 3 mois précédant l'abattage, leur poids a été contrôlé chaque semaine et leur état corporel chaque mois aux niveaux lombaire (L) et sternal (S), selon la méthode proposée par Morand-Fehr et al (1988). Le matin du jour de l'abattage, du sang jugulaire est prélevé pour doser les teneurs en acides gras non estérifiés (AGNE) et en glucose (GL). Les taux de lipides de l'organisme entier (\% LOE) et du tissu omental (\% LTO) sont calculés à partir des dosages d'extrait éthéré sur des échantillons broyés de la carcasse, du $5^{e}$ quartier et du grand et du petit épiploon.

Résultats et discussion - Le \% LOE moyen des 31 chèvres a été de $14,4 \%$ (écart type $\mathrm{ET}=7,0$ ) et de $11,5 \%$ $(\mathrm{ET}=5,0), 12,6 \%(\mathrm{ET}=5,3)$ et $19,4 \%$ $(E T=8,1)$ pour les chèvres abattues en début, en pleine lactation et taries, respectivement.

Les coefficients de corrélation entre $\%$ LOE et les différents paramètres explicatifs du \% LOE, et les écarts types résiduels, sont rapportés au tableau I. Les paramètres sanguins AGNE et $G L$, qui sont le reflet du métabolisme énergétique des animaux, ne présentent des corrélations significa- 
Tableau I. Corrélation entre le taux de lipides des chèvres et les paramètres sanguins, l'état corporel, le poids vif et le taux de lipides des tissus adipeux à l'abattage.

\begin{tabular}{lcccccc}
\hline Stade physiologique & $A G N E^{\mathrm{a}}$ & $G L^{\mathrm{a}}$ & $L^{\mathrm{a}}$ & $S^{\mathrm{a}}$ & $P V^{\mathrm{a}}$ & $\% \quad L T O$ \\
\hline Période sèche & $0,10^{\mathrm{No}}$ & $0,22^{\mathrm{N}}$ & $0,87^{++}$ & $0,87^{++}$ & $0,51^{\mathrm{N}}$ & $0,78^{++}$ \\
Début de lactation & $(8,5)^{\mathrm{C}}$ & $(6,7)$ & $(4,1)$ & $(4,2)$ & $(5,2)$ & $(5,5)$ \\
& $0,10^{\mathrm{N}}$ & $0,10^{\mathrm{N}}$ & $0,82^{++}$ & $0,81^{++}$ & $0,14^{\mathrm{N}}$ & $0,84^{++}$ \\
Pleine lactation & $(4,9)$ & $(4,9)$ & $(3,0)$ & $(3,1)$ & $(5,2)$ & $(2,8)$ \\
& $0,49^{\mathrm{N}}$ & $0,43^{\mathrm{N}}$ & $0,81^{++}$ & $0,83^{++}$ & $0,59^{+}$ & $0,81^{++}$ \\
Tous stades confondus & $(4,2)$ & $(3,7)$ & $(3,4)$ & $(3,3)$ & $(4,7)$ & $(2,2)$ \\
& $0,48^{++}$ & $0,45^{++}$ & $0,56^{++}$ & $0,68^{++}$ & $0,59^{++}$ & $0,71^{++}$ \\
& $(5,1)$ & $(5,2)$ & $(6,0)$ & $(5,3)$ & $(5,8)$ & $(5,1)$ \\
\hline
\end{tabular}

a $A G N E=$ Acides gras non estérifiés; $G L=$ glucose; $L=$ note lombaire; $S=$ note sternale; $P V=$ poids vif; ${ }^{b}$ coefficient de corrélation; $N=$ non significatif; $+=<0,05 ;++=P<0.01 ;{ }^{c}$ écant type résiduel en $\%$.

tives avec \% LOE que sur l'ensemble des 31 chèvres. En revanche, les corrélations entre le \% LOE, et les notes d'état corporel ou le taux de lipides du tissu omental sont hautement significatives mais elles sont plus faibles sur l'ensemble des 31 chèvres qu'à chaque stade! physiologique. Les corrélations avec les notes $S$ ou $L$ sont supérieures à 0,80 à chaque stade physiologique; mais sur l'ensemble des 31 chèvres, la corrélation avec $S$ est plus élevée qu'avec L. Les corrélations avec le poids vif sont significatives, excepté en début de lactation, ce qui peut s'expliquer par la part importante des variations de poids vif expliquée par l'évolution du poids des digesta en début de lactation. L'équation de régression en progression ascendante sur les 31 chèvres est la suivante: \% LOE $=21,7+6,2 \mathrm{~S}(\%)+0,16$ PV $(\mathrm{kg})$ $+7,0$ Index; $(R=0,85 \mathrm{ET}=3,9)$ si on affecte la valeur 1 à l'index pour les chèvres en lactation et 2 pour les taries.

Ainsi, il apparaît qu'à chaque stade physiologique, les notes d'état corporel peuvent être de bons estimateurs du \% LOE, meilleurs que le poids vif, quel que soit le stade physiologique, ce qui complète les premiers résultats obtenus sur chèvres (Morand-Fehr et al, 1990). Toutefois, les résultats sont moins bons, tous stades physiologiques confondus; un réajustement est nécessaire selon que les chèvres sont en lactation ou taries. Les valeurs des écarts types résiduels sont légèrement supérieures à celles de Rémond et al (1988) obtenues sur vaches par notation de l'état corporel. La teneur en lipides du tissu omental ne semble pas nettement être un meilleur prédicteur; de ce fait ce type de critère exigeant des biopsies est difficile à retenir. Enfin, les paramètres sanguins sont des médiocres estimateurs de \% LOE des chèvres.

Chilliard $\mathrm{Y}$, Rémond $\mathrm{B}$, Agabriel J, Robelin J, Vérité R (1987) Bull Tech CRZV INRA, Theix (70) 117-131

Morand-Fehr $P$, Bas $P$, Hervieu J, Sauvant D (1990) Reprod Nutr Dev 29 (suppl 2) 255s-256s

Morand-Fehr P, Hervieu J, Santucci $P$, Branca A (1988) CR Réunion du Groupe Agrimed (CEE), FAO, CIHEAM sur l'état corporel, Saragosse (Espagne) $\mathbf{1}^{\text {er }}$ Juin 1988

Rémond B, Robelin J, Chilliard Y (1988) Prod Anim 2, 111-114 